Original Paper http://ajol.info/index.php/ijbcs http://indexmedicus.afro.who.int

\title{
Etude sur la capacité de production, du circuit de commercialisation et de la consommation du zoom-koom vendu dans la ville de Ouagadougou au Burkina Faso
}

\author{
Adama Abdoul Razaak SOMA MASSIEKE ${ }^{1,2}$, Fidèle Wend-bénédo TAPSOBA ${ }^{2,3}$, \\ Donatien $\mathrm{KABORE}^{2 *}$, Issaka SEOGO${ }^{2}$, Abel TANKOANO ${ }^{2}, \mathrm{Mamoudou}$ Hama DICKO ${ }^{3}$, \\ Aboubacar TOGUYENI ${ }^{1}$ et Hagrétou SAWADOGO-LINGANI ${ }^{2}$ \\ ${ }^{1}$ Université Polytechnique de Bobo-Dioulasso, 01 BP.1091 Bobo-Dioulasso 01, \\ Tél. : (00226) 20980635 ; Fax : (00226) 20982527 ; Burkina Faso. \\ ${ }^{2}$ Département Technologie Alimentaire, Institut de Recherches en Sciences Appliquées et Technologies, \\ 03 BP. 7047 Ouagadougou 03.Tél:(00226) 25363786, Fax:(00226) 25363787 ; Burkina Faso. \\ ${ }^{3}$ Laboratoire de Biochimie Alimentaire, Enzymologie, Biotechnologie Industrielle et Bio-informatiques \\ (BAEBIB), Université Ouaga I Professeur Joseph KI-ZERBO, 03 BP 7021 Ouagadougou 03 ; Tel: +226 2530 \\ 7064 Burkina Faso. \\ *Auteur correspondant ; E-mail: kaboredonatien74@yahoo.fr
}

\section{REMERCIEMENTS}

Les remerciements vont à l'endroit du projet PAES-UEMOA/Burkina Faso (Projet d'Appui à l'Enseignement Supérieur) pour le soutien financier.

\section{RESUME}

La transformation des céréales comme le mil et le sorgho en zoom-koom n'associe pas toujours les bonnes pratiques d'hygiène et de fabrication (BPH/BPF). L'objectif principal de la présente étude est d'identifier les contraintes et les avantages liés à la production, consommation et commercialisation du zoomkoom. L'étude s'est déroulée dans la ville de Ouagadougou. Au total, 120 transformatrices (qui sont aussi les commerçants de zoom-koom) et 400 consommateurs de zoom-koom ont été enquêtés et les données ont été analysées par le logiciel CS Pro6 et Excel 2013. Les ingrédients les plus utilisés sont le gingembre (29\%) et la menthe (24\%). La majorité des transformatrices (70\%) utilisent entre 1 et $5 \mathrm{~kg}$ de mil pour produire le zoomkoom. De nombreuses transformatrices (97\%) affirment commercialiser elles-mêmes leur zoom-koom contre une minorité $(3 \%)$ qui affirment commercialiser leur zoom-koom dans les kiosques, les boutiques et les alimentations. Les sachets et les bidons plastiques récupérés sont les principaux emballages utilisés pour le conditionnement. Il ressort également que $70 \%$ des enquêtés consomment régulièrement le zoom-koom et $30 \%$ occasionnellement. Dans l'ensemble, le zoom-koom est beaucoup apprécié pour son goût mais $74 \%$ des consommateurs reconnaissent que sa qualité hygiénique peut être améliorée. Ainsi, des mesures doivent être prises afin de préserver la santé du consommateur et accroître sa valeur marchande.

(C) 2017 International Formulae Group. All rights reserved.

Mots clés: Zoom-koom, production, transformation, commercialisation, consommation. 


\title{
Study on the production capacity, marketing circuit and consumption of zoom-koom sold in the city of Ouagadougou in Burkina Faso
}

\begin{abstract}
The processing of cereals such as millet and sorghum in zoom-koom does not always combine good hygiene and manufacturing practices (GHP / GMP). The main objective of the present study is to identify the constraints and benefits associated with the production, consumption and commercialization of zoom-koom. The study was conducted in the city of Ouagadougou. A total of 120 processors (who are also zoom-koom traders) and 400 consumers of zoom-koom were surveyed and the data was analyzed by CS Pro6 and Excel 2013. The most commonly used ingredients are ginger (29\%) and mint (24\%). The majority of the transformers (70\%) used between 1 and $5 \mathrm{~kg}$ of millet to produce the zoom-koom. It appeared that $97 \%$ of transformers sell their zoom-koom themselves and only 3\% sell their zoom-koom in a market, kiosks, shops and food shop. Recycled plastic bags and bottles are used for packaging. $70 \%$ of consumers consume regularly zoom-koom and $30 \%$ occasionally. Overall, zoom-koom is much appreciated for its taste but $74 \%$ of consumers state that its hygienic quality should be improved. Thus, measures must be taken to safeguard the health of the consumers and increase its market value.
\end{abstract}

(C) 2017 International Formulae Group. All rights reserved.

Keywords: Zoom-koom, production, processing, commercialization, consumption.

\section{INTRODUCTION}

L'alimentation de rue est devenue un phénomène de la société moderne par sa grande importance socioéconomique permettant ainsi à toutes les catégories socioprofessionnelles de satisfaire à leurs besoins nutritionnels (Barro et al., 2002a). Les revenus générés par cette activité permettent à plusieurs ménages de couvrir les besoins financiers de la famille (Barro et al., 2007). Les aliments de rue sont reconnus être fréquemment associés à des maladies et plusieurs analyses microbiologiques ont révélé la présence de nombreuses bactéries pathogènes à des proportions dépassant les normes (Barro et al., 2007). Parmi les aliments de rue rencontrés à Ouagadougou, il y a des catégories d'aliments qui ont une dimension régionale. Il s'agit du Déguè, du Gonré, du Souma, du Benga, du Porc au four, des Brochettes et du Zoom-Koom (Barro et al., 2007).

Ces aliments résultent, la plupart du temps, de la transformation artisanale (traditionnelle) des produits agro-alimentaires. La technologie agro-alimentaire (traditionnelle) est caractérisée par des transformations manuelles d'aliments avec des opérations pénibles et peu hygiéniques pour mettre à la disposition des consommateurs une gamme de produits à base de céréales, de tubercules, de légumineuses, d'oléagineux, de fruits et légumes (Besadjo-Tchamba et al., 2014). Le constat est que peu de données existent sur les caractéristiques socioéconomiques liées à la transformation, la commercialisation et la consommation de ces aliments traditionnels à base de céréales comme le zoom-koom.

Le présent article a pour but de faire un état des lieux sur la capacité de production, le circuit de commercialisation et la consommation du zoom-koom vendu dans les douze (12) arrondissements de la ville de Ouagadougou au Burkina Faso.

\section{MATERIEL ET METHODES}

L'approche méthodologique adoptée dans la présente étude a consisté à l'identification des zones d'étude, à l'élaboration de l'outil de collecte des données, à la collecte des données sous forme d'enquête/entretien, à l'analyse et à l'exploitation de ces données collectées.

\section{Zone d'étude}

La présente étude est réalisée en janvier 2015 dans la ville de Ouagadougou (Burkina Faso) et a concerné les douze (12) arrondissements de la ville de Ouagadougou (Figure 1).

\section{Echantillonnage}


En absence de données statistiques nationales sur le nombre d'acteurs intervenant dans la production et la commercialisation du zoom-koom, la méthode d'échantillonnage empirique basée sur la fixation de quotas a été utilisée pour définir le nombre d'échantillons de productrices à enquêter dans la ville de Ouagadougou. A cet effet, cent vingt (120) productrices/commerçantes ont été retenues pour la présente étude. Pour ce qui concerne le nombre de consommateurs de zoom-koom, sur la base de l'enquête démographique de la ville de Ouagadougou réalisée en 2012, 400 consommateurs potentiels ont été retenus pour la présente étude.

\section{Outils de collecte}

Pour répondre aux objectifs de l'enquête, un questionnaire a été conçu pour les productrices et pour les commerçantes de zoom koom. Ce questionnaire est axé sur des indicateurs permettant de mesurer et/ou recueillir les points de vue des productrices et des commerçantes sur les matières premières et ingrédients utilisés dans la production du zoom-koom, sur les conditions de stockage, sur le conditionnement, le circuit de commercialisation, les prix du zoom-koom et la rentabilité du zoom-koom.

Un autre questionnaire a été élaboré à l'endroit des consommateurs afin de recueillir leur opinion sur la place de zoom-koom dans leur régime alimentaire. Ce questionnaire a aussi permis de recueillir l'appréciation des consommateurs sur le coût, le goût et la qualité du zoom koom.

\section{Collecte de données}

Quatre enquêteurs ont été retenus pour collecter les données après avoir suivi une formation sur l'administration des questionnaires et les bonnes conduites sur le terrain. Les enquêteurs ont été répartis dans les douze (12) arrondissements de la ville de Ouagadougou. La collecte des données a duré 21 jours.

\section{Traitement des données}

L'ensemble des données collectées ont été saisies et analysées en utilisant le logiciel CS Pro 6 et Excel 2013. Les tests statistiques ont porté essentiellement sur le test de Khi2.

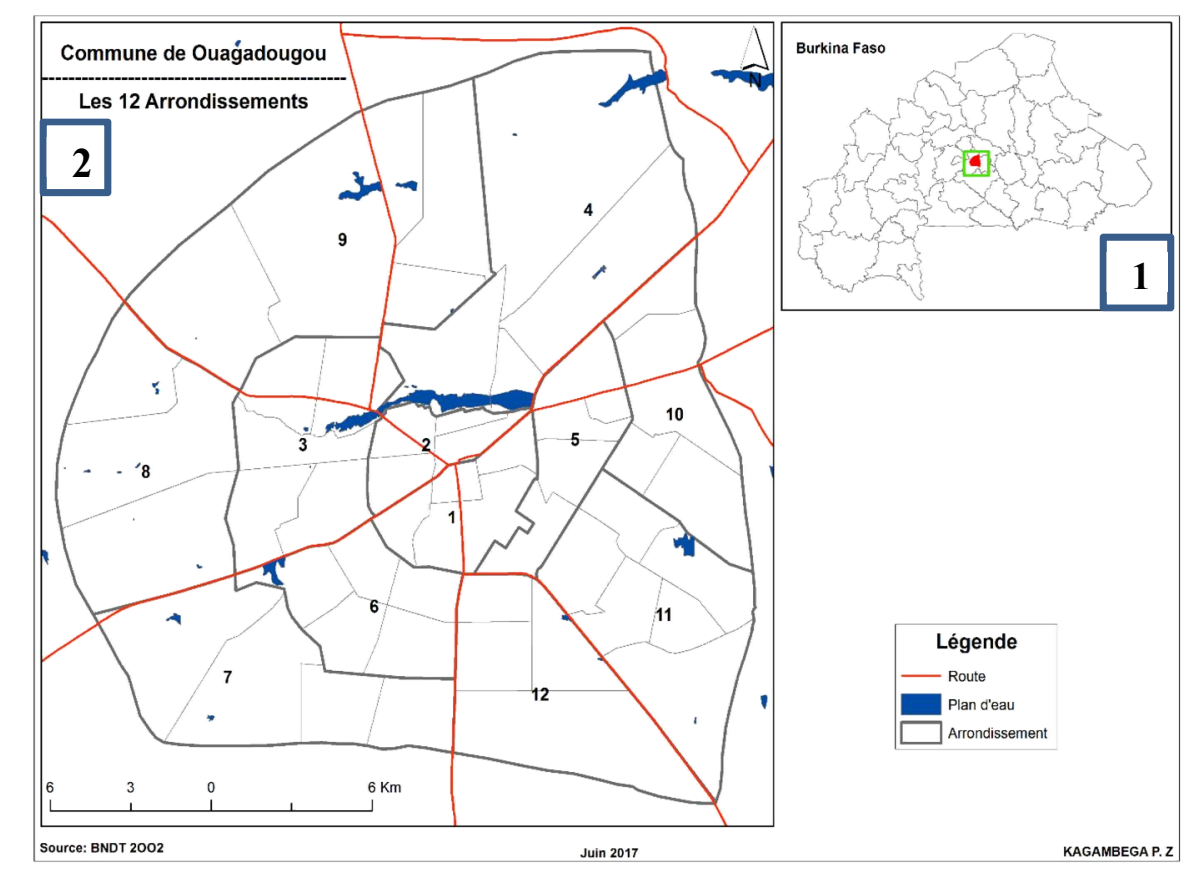

Figure 1: Carte du Burkina Faso présentant la situation géographique de Ouagadougou (1); ville de Ouagadougou illustrant les 12 arrondissements (2). 
RESULTATS

Typologie des personnes enquêtées

Les personnes enquêtées

(transformatrices, commerçantes et consommateurs de zoom-koom) avaient un âge compris entre $18-25$ ans $(30 \%)$; 26-35 ans $(35 \%) ; 36-45$ ans $(23 \%)$ et 45 ans et plus $(12 \%)$. La majorité de ces enquêtés étaient des femmes (53\%) (Tableau 1). Il faut noter aussi que la tranche d'âge des enquêtés compris entre 18 et 45 ans sont en majorité scolarisé et ont un niveau secondaire ou supérieur (Tableau 1). Par contre, ceux de la tranche d'âge compris entre 45 ans et plus sont pour la plupart analphabètes. Certains de cette catégorie d'âge ont au plus un niveau primaire. Les personnes ayant au moins un niveau secondaire ou supérieur consomment le zoom-koom régulièrement pour la plupart, tandis que celles qui ne sont pas alphabétisées ou ayant au plus un niveau primaire le consomment occasionnellement, surtout lors des cérémonies de baptêmes, de mariages et de bien d'autres occasions. Une minorité en consomme régulièrement.

Importance de la production du zoomkoom dans la ville de Ouagadougou

La production de zoom-koom est essentiellement artisanale et implique un grand nombre d'acteurs au Burkina Faso. Les enquêtes ont révélé que le mil (Pennisetum glaucum) est la principale matière première utilisée dans la production du zoom-koom et que le sorgho (Sorghum bicolor L. Moench) est rarement utilisé. La majorité des productrices l'obtiennent sur le marché et seulement $2 \%$ de ces transformatrices, cultivant du mil, utilisent leur production personnelle de mil. La cherté, l'indisponibilité et la mauvaise conservation des grains (mil) sont les principales difficultés liées à l'approvisionnement de la matière première. En plus du mil, d'autres ingrédients sont associés au processus de fabrication du zoomkoom. Au nombre de ces ingrédients tels que résumés dans la Figure 2, il y a le gingembre et la menthe qui sont les plus utilisés respectivement par $29 \%$ et $24 \%$ des transformatrices. Ensuite viennent respectivement le citron $(17 \%)$, le tamarin (16\%), l'arôme artificiel (9\%) et l'ananas $(5 \%)$.

Capacité des unités de production de zoomkoom en termes de quantités de matière première utilisée et de zoom-koom produit

Il ressort de cette étude que $80 \%$ des transformatrices utilisent entre 1 et $5 \mathrm{~kg}$ de mil, $13 \%$ qui utilisent 5 à $10 \mathrm{~kg}$ et $5 \%$ des transformatrices enquêtées utilisent 10 à $20 \mathrm{~kg}$ de mil (Figure 3). 82\% des transformatrices utilisant 1 à $5 \mathrm{~kg}$ de mil produisent entre 5 et 25 litres de zoom-koom par jour; 18\% utilisent la même quantité (1-5 kg) pour produire entre 25 et 50 litres de zoom-koom par jour. En moyenne, les transformatrices utilisent $1 \mathrm{~kg}$ de mil pour produire 10 litres de zoom-koom. Quant à la fréquence de production, $62 \%$ et $30 \%$ des transformatrices utilisant entre 1 à $5 \mathrm{~kg} \mathrm{du} \mathrm{mil,} \mathrm{produisent}$ respectivement chaque jour ou chaque deux jour le zoom-koom. Elles ne sont pas nombreuses, les productrices qui font le zoomkoom une fois par semaine $(3 \%)$ et deux fois par semaine $(5 \%)$.

Il ressort également des données recueillies que $33 \%, 40 \%$ et $27 \%$ des transformatrices utilisant 5 à $10 \mathrm{~kg}$ de mil (13\%) produisent respectivement 5-25 litres, 25-50 litres et 50-100 litres de zoom-koom. $73 \%$ des transformatrices utilisent 5 à $10 \mathrm{~kg}$ de mil pour produire le zoom-koom chaque jour et seulement $20 \%$ et $7 \%$ de ces transformatrices produisent respectivement le zoom-koom chaque deux jour et une fois par semaine. Les transformatrices utilisant une quantité de mil comprise entre 10 et $20 \mathrm{~kg}$ produisent 50 à 100 litres de zoom-koom chaque jour. Elles sont minoritaires (2\%), celles qui utilisent plus de $20 \mathrm{~kg}$ de mil pour produire 50 à 100 litres de zoom-koom par jour (Figure 4). Tous ces résultats dénotent du caractère artisanal de la production du zoom- 
koom. En somme, on peut retenir que le zoomkoom se produit quotidiennement pour la plupart des transformatrices et la capacité de production varie d'une unité artisanale à une autre.

\section{Conditionnement et stockage du zoom- koom}

Les emballages les plus utilisés par les productrices pour le conditionnement du zoom-koom sont les sachets plastiques (30\%), les petites bouteilles de 0,5 litre $(35 \%)$ et les grandes bouteilles plastiques de récupération de 1 et 1,5 litres (35\%) (Figure 5). Après le conditionnement, le zoom-koom conditionné est conservé soit dans un réfrigérateur, un congélateur, une glacière avec ou sans la glace, ou dans un sceau. Les résultats montrent également que la majorité (83\%) des répondants sont de petites productrices à telle enseigne que le zoom-koom produit ne peut excéder un jour de commercialisation.

Environ $30 \%$ des personnes enquêtées ont constaté des altérations de leur zoom-koom pouvant être dues, selon elles, à la fermentation du produit. Les causes de ces altérations varient selon les répondants. Il s'agirait, entre autres, du délestage d'électricité, des pannes de réfrigérateurs, de l'exposition du zoom-koom au soleil pendant une longue durée, du manque de conservateur ou du dosage très élevé du tamarin.

\section{Commercialisation du zoom-koom dans la ville de Ouagadougou \\ Circuit de commercialisation du zoom-koom}

La majorité des transformatrices (95\%) produisent et commercialisent du zoom-koom à base de mil. Seulement $5 \%$ produisent et commercialisent du zoom-koom à base de sorgho. Le circuit de vente /distribution du zoom-koom n'est pas bien développé dans la ville de Ouagadougou. Ces lieux de vente sont principalement des boutiques/kiosques (10\%), des alimentations (2\%), des marchés/yaar $(87 \%)$ et de la production familiale pour la consommation (1\%). Le zoom-koom peut s'obtenir également auprès des productrices et vendeurs ambulants.

\section{Prix du zoom-koom conditionné}

Le zoom-koom est vendu dans des sachets plastiques et dans des bouteilles plastiques (petit et grand format). $71 \%$ des répondants vendent le zoom-koom conditionné dans les sachets plastiques de $30 \mathrm{cl}$ à 25 FCFA contre $29 \%$ qui le commercialisent dans des sachets de $50 \mathrm{cl}$ à 200 FCFA avec un prix moyen de 114 FCFA. Il ressort que $36 \%$ des consommateurs enquêtés achètent le zoomkoom conditionné dans les bidons d'un demilitre au prix moyen de 142 FCFA. Environ $16 \%$ des répondants l'achètent dans les bidons de 1,5 litre au prix moyen de 520 FCFA. Ils sont $24 \%$ à consommer le zoom-koom vendu en vrac dans des verres/gobelets/bols plastiques de $30 \mathrm{cl}$ au prix moyen de 78 FCFA. Seulement, $8 \%$ des consommateurs enquêtés achètent le zoom-koom avec le bidon de 1 litre au prix moyen de 414 FCFA (Figure 6). Au regard de ces résultats, il ressort que le prix d'un (1) litre de zoom-koom vendu à Ouagadougou est d'environ 350 FCFA.

\section{Recettes de la commercialisation du zoom- koom}

Il ressort des données collectées que $53 \%$ des transformatrices produisant du zoomkoom à partir d'une quantité de mil comprise entre 5-10 kg ont révélé avoir une recette journalière variant entre 2000 et 5000 FCFA. Seulement $28 \%, 12 \%$ et $7 \%$ des vendeuses ont des recettes journalières se situant respectivement dans les intervalles de 1000 à 2000 FCFA (utilisant $1-5 \mathrm{~kg}$ de mil), de 5000 à 10000 FCFA (utilisant $10-20 \mathrm{~kg}$ de mil) et de plus de 10000 FCFA (utilisant plus de 20 $\mathrm{kg}$ de mil) (Figure 7). Sur un plan d'ensemble, les différentes transformatrices utilisant une quantité de mil comprise entre $1-5 \mathrm{~kg} ; 5-10$ $\mathrm{kg} ; 10-20 \mathrm{~kg}$ et plus de $20 \mathrm{~kg}$ font une recette mensuelle respectivement de $45000 ; 105$ $000 ; 225000$ et plus de 300000 FCFA en moyenne. Sur un coût total (1 650 FCFA) de transformation de $1 \mathrm{~kg}$ de mil en zoom-koom, 
les transformatrices réaliseraient un bénéfice journalier de 350 FCFA et 10500 FCFA mensuel. Pour un coût total (4 000 FCFA) de transformation de $5 \mathrm{~kg}$ de mil en zoom-koom, les transformatrices réaliseraient un bénéfice journalier de 1000 FCFA et 30000 FCFA mensuel. Il faut dire que plus la quantité de production augmente plus le montant des bénéfices augmentent.

Dans l'ensemble, les acteurs de la commercialisation ont dit être satisfaits de leur activité. Sur un total de 120 commerçants, $39 \%$ ont estimé que la commercialisation du zoom-koom est peu rentable ; $37 \%$ pensent qu'elle est rentable et $23 \%$ pensent qu'elle est moyennement rentable. Seulement $1 \%$ des répondants affirment que la commercialisation du zoomkoom n'est pas du tout rentable.

Appréciations de la qualité et de la fréquence de consommation du zoom-koom

Les consommateurs de zoom-koom sont de tout âge (enfants, jeunes, adultes et personnes âgées) et de tout sexe (féminin et masculin). Toutes les personnes enquêtées ont répondu avoir déjà consommé le zoom-koom. Il est ressortit des résultats que 32,5\% des répondants consomment le zoom-koom une fois par semaine, $9,5 \%$ chaque jour, $26 \%$ chaque deux jour et $30 \%$ occasionnellement, surtout aux moments des cérémonies de baptêmes, de mariages et pendant les périodes des fêtes (Tableau 2). La majorité (73\%) des consommateurs donnent des appréciations positives sur le goût du zoom-koom. En effet, ils sont $73 \%$ à déclarer que le zoom-koom a un bon goût contre $5 \%$ qui affirment le contraire (Tableau 2). Environ 22\% des consommateurs jugent que son goût est acceptable. Selon l'avis de certains consommateurs, l'ajout de produits chimiques dans le zoom-koom, le manque d'hygiène, l'utilisation excessive de sucre et de la farine contribuaient à la détérioration de la qualité du zoom-koom. Malgré l'appréciation positive aussi bien du goût et de la qualité du zoom-koom, 74\% des consommateurs pensent que le zoom-koom n'est pas produit dans des bonnes conditions hygiéniques (Tableau 2). Seulement, $21 \%$ et $5 \%$ ont trouvé que les conditions de production du zoom-koom sont acceptables et bonnes, respectivement (Tableau 2). Les consommateurs ont estimé que le zoom-koom produit dans des conditions douteuses peut provoquer des maladies telles que les maux de ventre, la diarrhée et le choléra. L'ensemble des consommateurs interrogés pensent que la qualité du zoom-koom peut être améliorée à travers la sensibilisation des transformatrices de zoom-koom et au renforcement de capacités de ces dernières.

Tableau 1 : Typologie des acteurs de la production du zoom-koom.

\begin{tabular}{|c|c|c|c|c|c|c|}
\hline \multirow{2}{*}{$\begin{array}{l}\text { Caractéristiques socio- } \\
\text { démographiques }\end{array}$} & \multicolumn{4}{|c|}{ Age (ans) } & \multicolumn{2}{|c|}{ sexe } \\
\hline & 18-25 & 26-35 & $36-45$ & +45 & Homme & Femme \\
\hline $\begin{array}{l}\text { Proportion des répondants } \\
(\%)\end{array}$ & 30 & 35 & 23 & 12 & 47 & 53 \\
\hline Niveau de scolarisation & $\begin{array}{c}\text { Universitaire } \\
\text { et } \\
\text { secondaire }\end{array}$ & $\begin{array}{c}\text { Universitaire } \\
\text { et } \\
\text { secondaire }\end{array}$ & $\begin{array}{c}\text { Universitaire } \\
\text { et } \\
\text { secondaire }\end{array}$ & Primaire & & \\
\hline $\begin{array}{l}\text { Consommation du zoom- } \\
\text { koom }\end{array}$ & Habituelle & Habituelle & Habituelle & Occasionnelle & & \\
\hline
\end{tabular}




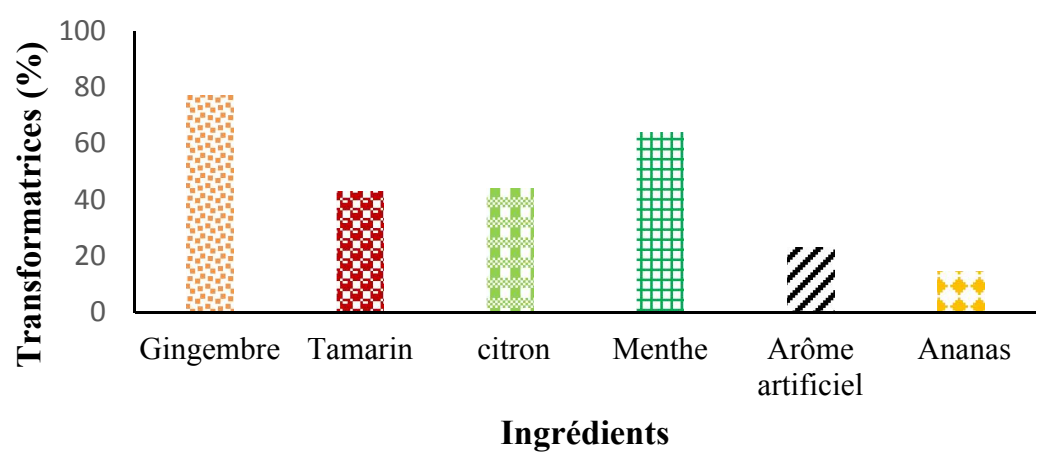

Figure 2: Répartition des transformateurs en fonction des ingrédients utilisés dans de la production du zoom-koom.

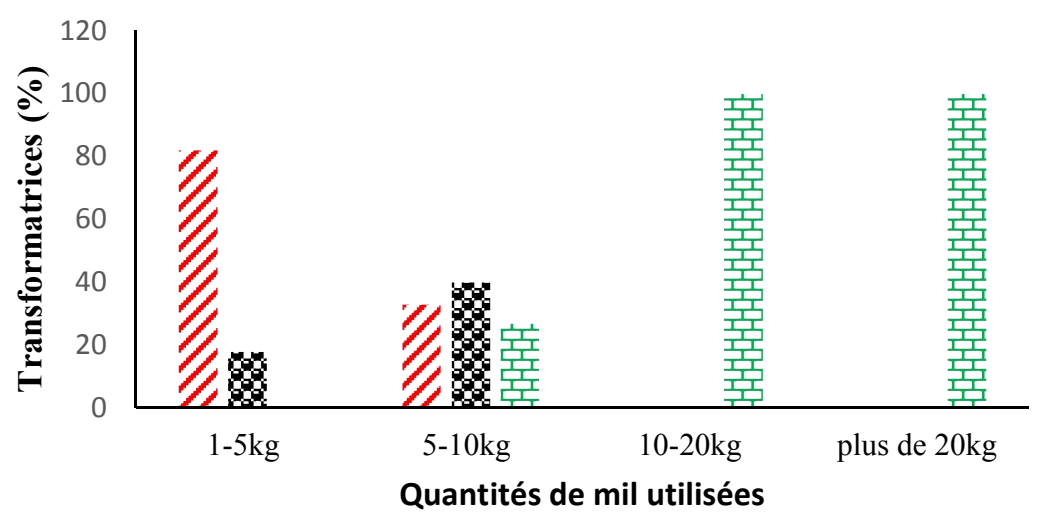

$\rightarrow 5-25 \mathrm{~L} \quad x 25-50 \mathrm{~L}=50-100 \mathrm{~L}$

Figure 3: Quantité de mil utilisée et de zoom-koom produit dans la production du zoomkoom.

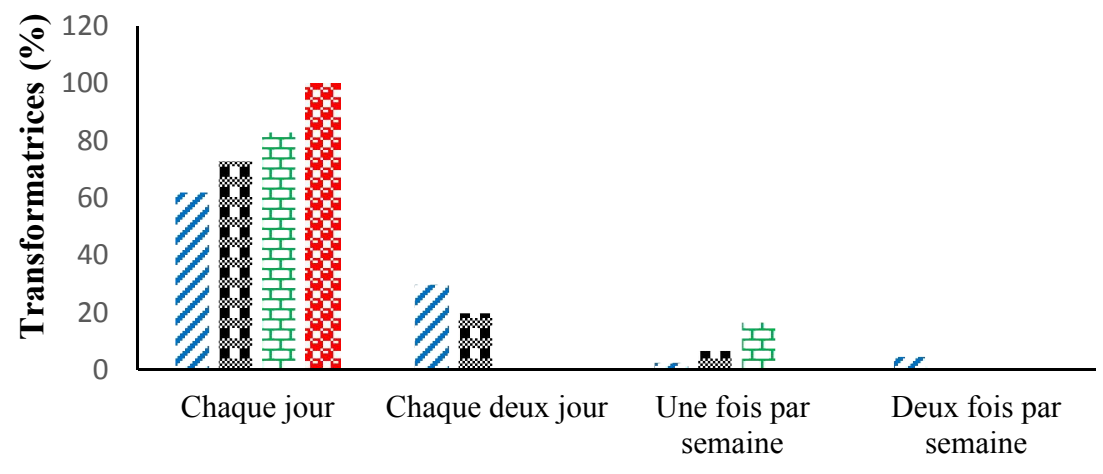

Fréquence de production

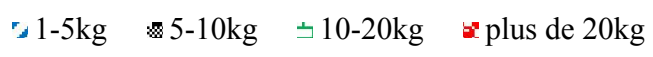

Figure 4: Quantité de mil utilisée et fréquence de production du zoom-koom. 


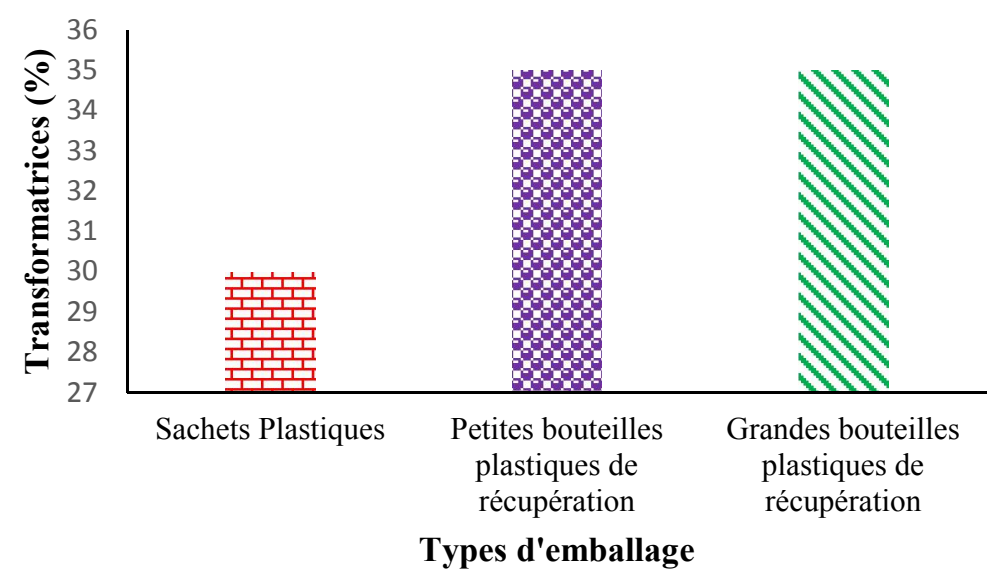

Figure 5: Types d'emballages utilisés pour le conditionnement du zoom-koom.

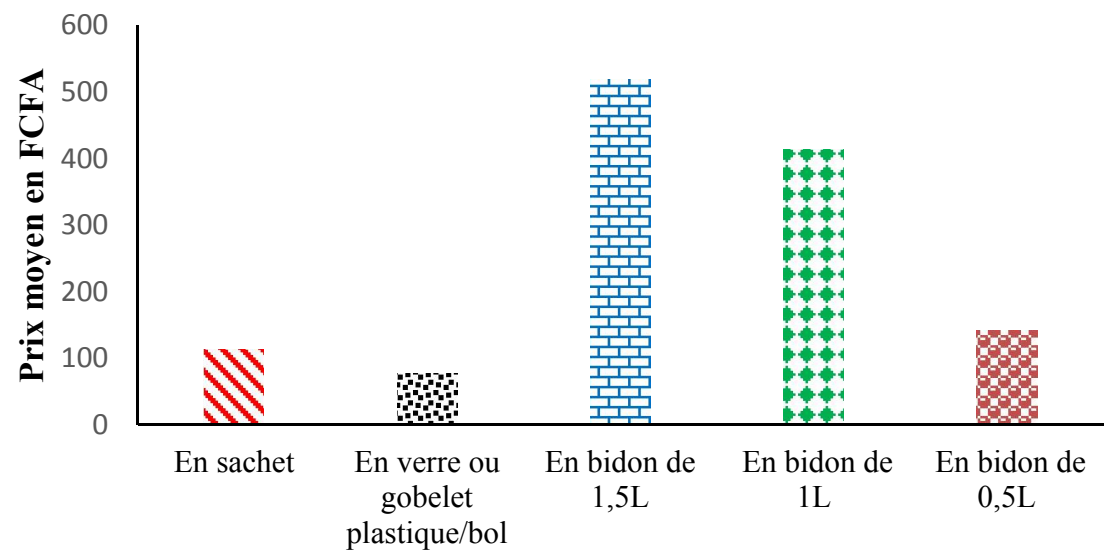

Types de conditionnement

Figure 6: Prix moyen du zoom-koom en fonction du type de conditionnement.

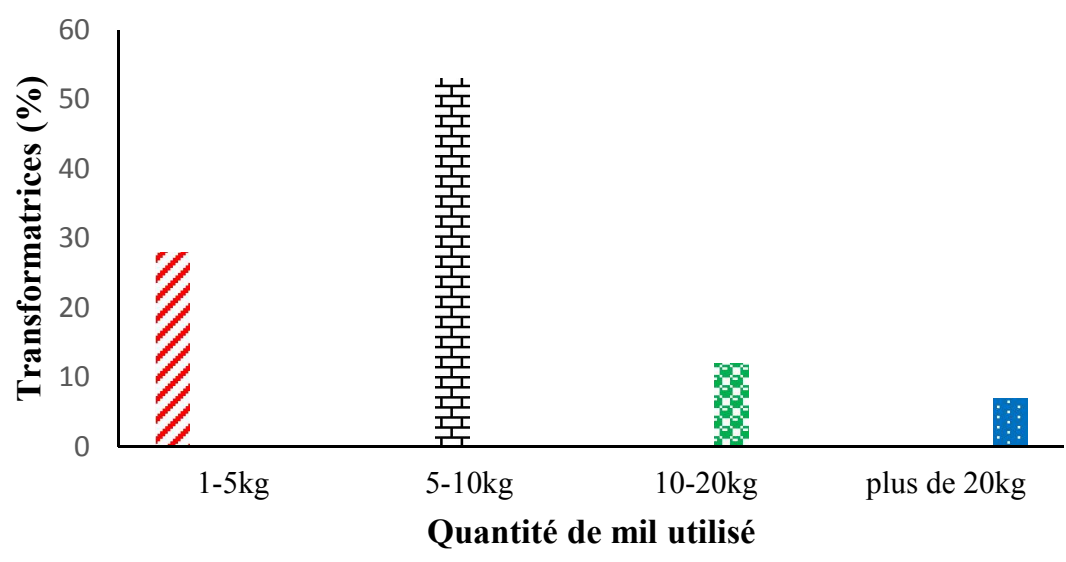

$$
\because 1000-2000 \quad \text { 工2000-5000 5000-10000 घplus de } 10000
$$

Figure 7: Rentabilité du zoom-koom en fonction du pourcentage des répondants. 
Tableau 2 : Appréciation de la qualité et des conditions de production du zoom-koom

\begin{tabular}{ccccccc}
\hline & \multicolumn{3}{c}{ Goût } & \multicolumn{2}{c}{ Conditions de production } \\
\cline { 2 - 6 } & Bon & Acceptable & Mauvais & Bonne & Acceptable & Mauvaise \\
\hline $\begin{array}{c}\text { Proportion des } \\
\text { répondants (\%) }\end{array}$ & 73 & 22 & 5 & 5 & 21 & 74 \\
\hline $\begin{array}{c}\text { Fréquence de } \\
\text { consommation }\end{array}$ & Chaque jour & Chaque deux jours & $\begin{array}{c}1 \text { fois } \\
\text { /semaine }\end{array}$ & Occasionnelle \\
\hline $\begin{array}{c}\text { Proportion des } \\
\text { répondants (\%) }\end{array}$ & 29,5 & \multicolumn{2}{c}{26} & 32,5 & \multicolumn{2}{c}{12} \\
\hline
\end{tabular}

\section{DISCUSSION}

Le mil est la céréale la plus utilisée pour la production du zoom-koom comparativement au sorgho. La faible utilisation du sorgho dans la production du zoom-koom pourrait être due à sa composition. En effet, le sorgho étant très riche en tanins, ces composés phénoliques qui, d'un côté, se sont montrés capables de protéger les grains contre les attaques fongiques, les insectes et les oiseaux ce qui, sans doute, est un avantage agronomique réduisent la digestibilité enzymatique aussi bien des protéines que des amidons et autres polysaccharides (Tawaba et al., 2013). Cependant, le nettoyage des grains de sorgho, le trempage, le tamisage et la fermentation sont des opérations qui réduisent de façon significative la teneur en phénols totaux et en anthocyanes dans la bouillie dérivée de sorgho (Kayodé et al., 2012). L'ajout de gingembre et de la menthe (ingrédients les plus utilisés) est plus orienté dans la recherche de leurs arômes et de la sensation de goût piquant pour le gingembre. Le gingembre présente un avantage sur le plan thérapeutique car il contiendrait des antioxydants qui pourraient prévenir les maladies cardiovasculaires, certains cancers et d'autres maladies liées au vieillissement (Eustache, 2009). Le gingembre facilite également la digestion et exerce un effet antiémétique, c'est-à-dire qu'il peut aider à lutter contre les nausées et les vomissements
(Bassolé, 2003; Abessolo, 2004). La menthe verte, quant à elle, contient des huiles essentielles, des flavonoïdes, des acidesphénols et du menthol d'où son puissant arôme (Ginseng, 2013). En plus de son arôme, la menthe contient du potassium, et de l'acide folique ou Vitamine B9 (Ginseng, 2013). L'ajout du tamarin est plus pour son caractère acidifiant que pour son arôme. Le zoom-koom est une boisson qui contient des oligoéléments qui jouent un rôle très important dans l'alimentation humaine en général et des enfants en particulier (Soma, 2014). Ces oligo-éléments participent à la formation et à la solidification des os chez l'enfant (Ca et $\mathrm{P}$ ), à la formation du sang ( $\mathrm{Fe}$ et $\mathrm{Mg}$ ) et surtout à lutter contre les anémies fréquents chez les enfants en bas âges et les femmes en période de grossesse (Soma, 2014).

Le caractère artisanal de l'activité de la transformation du mil en zoom-koom fait que les femmes n'utilisent pas d'emballages appropriés pour le conditionnement de cette boisson. Il s'agit le plus souvent des bouteilles récupérées et des sachets plastiques qu'elles utilisent pour le conditionnement. Le prix du zoom-koom est fonction de la taille de l'unité de transformation, de la diversité des ingrédients utilisés, du type de conditionnement et du lieu de vente/distribution. L'accessibilité de cette boisson à un grand nombre de consommateurs 
fait que cette activité est rentable et les femmes arrivent à subvenir à leurs besoins.

Cependant, la capacité de production du zoom-koom par les unités de transformations enquêtées est faible au regard de la quantité de mil transformé. Cela dénote de la nature artisanale de cette activité. La grande majorité des transformatrices produisent peu en fonction de l'écoulement quotidien et du fait que le produit fini n'est pas stabilisé, donc difficile à conserver. En effet, lorsque les aliments non stabilisés sont exposés pendant de longues heures au cours de la vente dans de mauvaises conditions de température, ils deviennent vulnérables à la contamination par plusieurs types de microorganismes (Barro et al., 2007) et s'altèrent. Après la contamination, les microorganismes s'y développent rapidement affectant la qualité microbiologique de l'aliment. En effet, Besadjo-Tchamba et al., (2014) ont également identifié la présence de microorganismes pathogènes tels que Salmonnella spp et E. coli (EPEC) dans le zoom-koom, dont la présence seraient due probablement au manque d'hygiène au cours $\mathrm{du}$ processus de transformation du mil en zoom-koom et également lors des opérations de vente et de conditionnement.

Pour éviter cela, les aliments de rue y compris les boissons doivent être bien conservés et manipulés en respectant les bonne pratiques d'hygiène $(\mathrm{BPH})$ au cours des opérations de vente pour préserver leur qualité (Nout et al., 2003b) et la santé du consommateur. Certaines transformatrices utilisent peu de mil pour produire une grande quantité de zoom-koom, afin d'augmenter les recettes journalières. Cela a un impact sur la qualité organoleptique du produit fini. Dans ces conditions, le zoom-koom est fortement dilué, devient moins consistant (contient peu de matière sèche), donc moins apprécié des consommateurs. Le zoom-koom est produit presque chaque jour par la majorité des transformatrices enquêtées. $\mathrm{Ce}$ constat pourrait s'expliqué par le fait que c'est une activité génératrice de revenus pour ces femmes. En effet, les revenus générés par cette activité permettent à plusieurs ménages de couvrir les besoins financiers de la famille (Canet et N'Diaye, 1996; Mwangi, 2002; Barro et al., 2002b; Ohiokpehai, 2003). Le zoom-koom est beaucoup consommé et apprécié du public, car comme d'autres aliments de rue, cette boisson de rue permet à toutes les catégories socioprofessionnelles de satisfaire à leurs besoins nutritionnels (Barro et al., 2002a; Dawson et Canet, 1991).

Cependant, le zoom-koom étant un produit dont la technologie n'inclut pas une étape de traitement thermique, n'est pas stabilisé au plan microbiologique et reste donc de qualité douteuse. En effet, selon Barro et al. (2007), le zoom-koom est l'aliment de rue qui renferme le plus de coliformes thermotolérants et totaux. Les aliments de rue sont reconnus être fréquemment associés à des maladies et plusieurs travaux antérieurs ont révélé la présence de nombreuses bactéries pathogènes à des nombres dépassant les normes (King et al., 2000; Barro et al., 2002a; Barro et Traoré, 2004). Ces données microbiologiques montrent que les aliments de rue sont sujets à un problème de salubrité lors de la préparation, de la conservation et pendant la vente (Bryan et al., 1992).

\section{Conclusion}

Le mil est la principale matière première généralement utilisée pour la production du zoom-koom. Le procédé de transformation de mil en zoom-koom est essentiellement artisanal. L'emballage utilisé pour le conditionnement est essentiellement constitué par des sachets et bouteilles plastiques récupérés. Les productrices de zoom-koom font aussi la commercialisation. Cependant, le circuit de distribution est peu développé. Les consommateurs du zoom-koom 
sont de tout âge et de toutes classes sociales. Malgré l'appréciation positive de la qualité organoleptique du zoom-koom, les consommateurs sont majoritaires à penser que le zoom-koom n'est pas produit dans de bonnes conditions hygiéniques. Pour améliorer la qualité hygiénique du zoomkoom, celui-ci doit être bien conservé et manipulé en respectant les bonnes pratiques d'hygiène (BPH) et de fabrication (BPF) au cours des opérations de transformation et de vente. Pour cela, une sensibilisation sur les bonnes pratiques et un renforcement de capacité des acteurs est nécessaire.

\section{CONFLITS D'INTERETS}

Les auteurs déclarent n'avoir aucun conflit d'intérêts quant à la réalisation de cette étude et à la rédaction de cet article.

\section{CONTRIBUTIONS DES AUTEURS}

Tous les auteurs ont participé à la collecte, au traitement des données et à la rédaction de cet article.

\section{REMERCIEMENTS}

Les remerciements vont à l'endroit des techniciens du Département Technologie Alimentaire (DTA) de l'IRSAT pour leur collaboration.

\section{REFERENCES}

Abessolo AN. 2004. Etudes de deux plantes aromatiques d'utilisation courante au Burkina Faso: Mentha piperita L. et Cymbopogon citratus (D.C.) Stapf Mémoire de fin d'études / Diplôme d'Études Supérieures Spécialisées (D.E.S.S.) / UO. Burkina Faso, p.47.

Barro N, Ouattara CAT, Nikiéma AP, Ouattara AS, Traoré SA. 2002a. Evaluation de la qualité microbiologique de quelques aliments de rue dans la ville de Ouagadougou au Burkina Faso. Cahier Santé , 12(4): 369-74.
Barro N, Nikiéma P, Ouattara CAT, Traoré AS. 2002b. Evaluation de l'hygiène et de la qualité microbiologique de quelques aliments rue et les caractéristiques des consommateurs dans les villes de Ouagadougou et de Bobo-Dioulasso (Burkina Faso). Rev. Sci Tech. Sci. Santé, 25: 7-21.

Barro N, Traoré SA. 2004. Identification des aliments de rue de grande consommation nécessitant un suivi de contrôle de qualité. Rapport d'études sur les aliments de rue à Ouagadougou CRSBA, p.24.

Barro N, Ouédraogo O, Abdoul BR, Nikiema PA, Ilboudo AJ, Ouattara AS, Ouattara CAT \& Traoré AS. 2007. Impact de la température de vente sur l'altération de la qualité microbiologique de quelques aliments de rue à Ouagadougou (Burkina Faso). Journal des Sciences, 7(2): 25-32.

Bassolé IHN. 2003. Composition chimique et activités antimicrobiennes, ovocides et larvicides des huiles essentielles des feuilles et fleurs de Cymbopogon proximus (stapf.), Lippia chevaleri (Mold.), (Mold.) et Ocimum canum (sims), Thèse unique en biochimie et microbiologie ; UFR-SVT; Université de Ouagadougou. p.3-10.

Bryan FL, Teufel P, Riaz S, Rooth S, Qadar F, Malik Z. 1992. Hazards and critical control points of street-vended chat, a regionally popular food in Pakistan. $J$ Food Protec., 55(9): 708-713. DOI: https://doi.org/10.4315/0362-028X55.9.708

Besadjo-Tchamba G, Bawa IH, Nzouankeu A, Bagré TS, Dembélé R, Bonkoungou IJO, Zongo C, Savadogo A, Traoré AS, Barro N. 2014. Occurrence and antimicrobial susceptibility of Escherichia coli and Salmonella spp. isolated from "zoomkoom" beverage and ice in Ouagadougou, Burkina Faso. African Journal of Microbiology Research, 
8(35): 3243-3249. DOI: $10.5897 /$ AJMR2014.7014.

Canet C, N'Diaye C. 1991. L'alimentation de rue en Afrique $F N A / A N A$ 17/18, 1996; 413.Dawson RJ, Canet C. International activities in street food. Food contr., 2: 135-139.

Canet C, N'Diaye C. 1996. L'alimentation de rue en Afrique FNA/ANA 17/18. p.4-13.

El-Scherbeeny MR, Saddik MF, Aly HES, Bryan FL. 1985. Microbial profiles and storage temperatures of Egyptian rice dishes. J. Food Protec., 48: 39-43.

Eustache I. 2009. Chimiothérapie : contre les nausées, pensez au gingembre ; $45^{\text {ème }}$ congrès de l'American Society of Clinical Oncology (ASCO); Orlando, p.2.

Ginseng CAM. 2013. Le gingembre, propriétés, bienfaits, posologie, effets secondaires. Ginseng CAM ; p.41.

Kayodé APP, Akogou FUG, Amoussa Hounkpatin W et Hounhouigan DJ. 2012. Effets des procédés de transformation sur la valeur nutritionnelle des formulations de bouillies de complément à base de sorgho. Int. J. Biol. Chem. Sci., 6(5): 2192-2201. DOI: http://dx.doi.org/ 10.4314/ijbcs.v6i5.25

King LK, Awumbila B, Canacoo EA, OfosuAmaah S. 2000. An assessment of the safety of street foods in the Ga district of Ghana; implication for the spread of zoososes. Acta Tropica., 76: 39-43.
Mwangi A. 2002. Nutritional, hygenic and socioeconomic dimensions of street foods in urban areas: The case of Nairobi. Wageningen University Dissertation; $\mathrm{N}^{\circ}$ 3157. March (Abstract).

Nout R, Hounhouigan J, van Boekel T. 2003b. Composants principaux et propriétés des aliments. In Les Aliments Transformation, Conservation et Qualité, Nout R, Hounhouigan J, van Boekel T (eds). Backhuys Publishers \& CTA co-publication: Wageningen, Leiden, Netherlands ; 1-13.

Ohiokpehai O. 2003. Nutritionnal aspects of street foods in Botswana. Pakistan $J$ Nutr., 2: 76-81.

Soma MAAR. 2014. Utilisation de cultures de Lactobacillus fermentum dans la technologie du zoom-koom, une boisson locale à base de mil (pennisetum glaucum) pour améliorer sa qualité nutritionnelle, sanitaire et organoleptique. Mémoire de fin d'études, en vue de l'obtention du master en biologie appliquée et modélisation des systèmes biologiques. Institut du Développement Rural (IDR), p.1-85.

Tawaba JCB, Ba K, Destain J, Malumba P, Béra F, Thonart P. 2013. Vers une intégration $\mathrm{du}$ sorgho comme matière première pour la brasserie moderne (synthèse bibliographique). Biotechnol. Agron. Soc. Environ., 17(4): 622-633.

Umoh VJ, Odoba MB. 1999. Safety and quality evaluation of street foods sold in Zaria, Nigeria. Food Contr., 10: 9-14. 